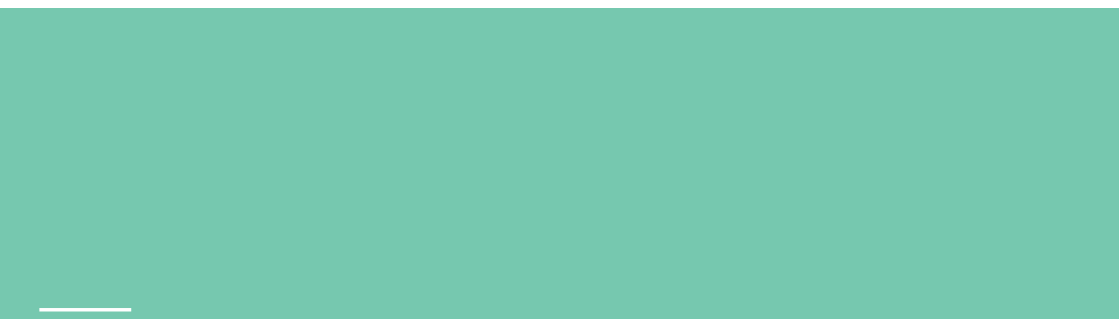

\title{
Valparaíso, puerto y montaña: un desafío [en] pendiente.
}

- Palabras clave/ Planificación, sustentabilidad, vivienda, Valparaíso, Melbourne.

A Keywords/ Planning, sustainability, dwelling.

Valparaíso, Melbourne.

A Recepción/ 17 junio 2014

A Aceptación/ 27 agosto 2014

\section{Valparaíso, Port and Mountain: Challenge} on a Slope.

\section{Dra. Beatriz Maturana Cossio}

PhD, University of Melbourne, Australia.

Master of Urban Design, University of

Melbourne, Australia.

Bachelor of Architecture, Instituto Real de Tecnología de Melbourne, Australia.

Académica, Instituto de la Vivienda, Facultad de Arquitectura y Urbanismo, Universidad de Chile. maturana@uchilefau.cl

RESUMEN/ Las siguientes reflexiones se enmarcan en un seminario presentado durante la Semana de la Sustentabilidad (mayo, 2014), en la Universidad de Chile. Este seminario se enfocó en el reciente incendio de Valparaíso, el que se contrasta con el incendio que afectó parte del estado de Victoria, Australia (febrero 7, 2009). Ambos incendios se consideran los más devastadores en la historia de estos países. Para este seminario se sugirió responder a cuatro preguntas, las que ayudaron a orientar la presentación y también a cuestionar algunas de las premisas. Entre estas premisas está la idea de que se puede solucionar un problema de escala urbana a través del objeto vivienda, lo que conlleva a un uso distorsionado de la noción de sustentabilidad. Entre las similitudes y diferencias de estos dos casos, llama la atención que en ambos se enfatizan las soluciones de vivienda como respuesta a un problema mucho más crítico, que era y sigue siendo, la falta de planificación. ABSTRACT/ The following reflections are part of a seminar presented during the Sustainability Week (May, 2014), in the University of Chile. The seminar addressed the recent fire in Valparaíso, comparing it with the fire that affected part of the state of Victoria, Australia (February 7, 2009). Both fires are considered to be the most devastating events in the history of each country. The seminar worked with four questions, which helped guide the presentation and questioned certain premises. One of these premises was the notion that an urban scale issue can be solved at the housing level, which is a distorted use of the idea of sustainability. Comparing both cases, a notable issue is that both put an emphasis on housing solutions to address a much more critical problem, which was and still is a lack of planning.

Las preguntas formuladas por los organizadores del seminario de la "Semana de la Sustentabilidad", organizada por la Universidad de Chile y que discutiré a continuación son: ¿En qué consiste la arquitectura, construcción y/o urbanismo sustentable? ¿A qué tipo de construcciones se puede aplicar? ¿Cómo se puede ejecutar (requerimientos, condiciones, estrategias)? y ¿̇cuáles son sus ventajas y dificultades?

\section{¿EN QUÉ CONSISTE LA \\ ARQUITECTURA, CONSTRUCCIÓN Y/O URBANISMO SUSTENTABLE?}

Este es un tema extenso, pero me enfocaré al concepto central -la noción de sustentabilidad - o sea, el sostenimiento o mantención (en este caso) de las condiciones previas a la tragedia. La respuesta es clara. No se pueden mantener las mismas condiciones que generaron este y otros incendios, los que ya nos estaban alertando de una situación de emergencia. Es importante notar que tanto en el incendio de Victoria, Australia, como en el de Valparaíso, Chile, muchos de los damnificados ya habían sido víctimas de previos incendios. Sólo un año atrás un incendio había destruido 35 viviendas y 40 hectáreas en los cerros Mariposas y La Cruz en Valparaíso (Valencia, 2014). Por lo tanto, más allá de enfrentar el día a día, no necesitamos ser expertos en la materia para afirmar que se necesitan cambios y mejoras (sociales, medioambientales y económicas) que fomenten la sustentabilidad de la ciudad en el largo plazo. Esto pasa por reconocer la enorme inequidad medioambiental característica de Valparaíso, situación exacerbada por una expansión urbana sin control, con muchas viviendas que, sin ser necesariamente malas, se emplazan en sectores de alto riesgo y como afirma Sebastián Grey (presidente del Colegio de Arquitectos), "pariendo una geografía caótica que no aguanta a los vehículos de emergencia" (Sepúlveda, 2014). En estos últimos meses hemos sido testigos de una comunidad local, profesional y política que exige cambios y la implementación de planificación urbana (MINVU, 2014; Valencia, 2014). 


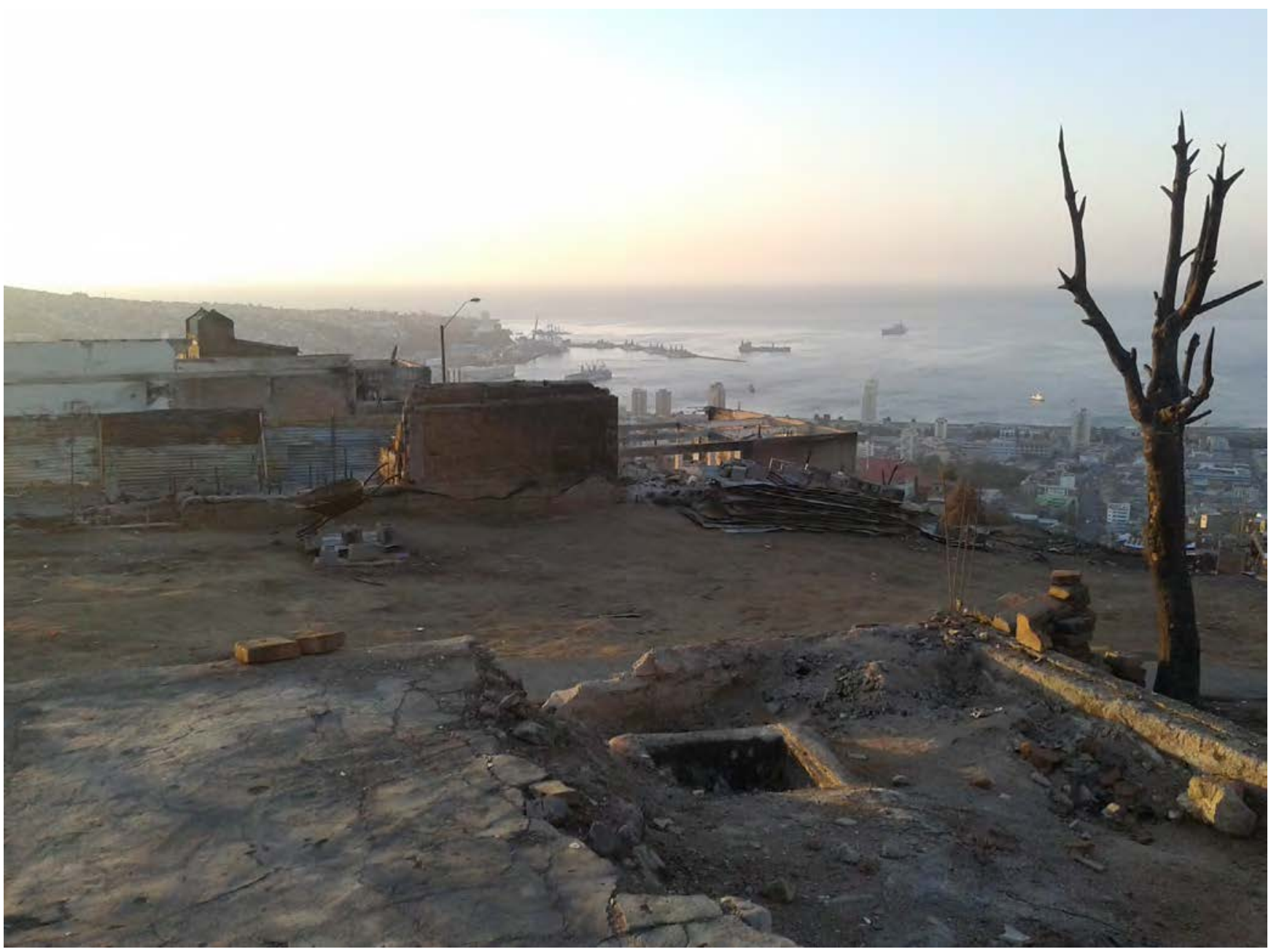

Imagen 1. El puerto de Valparaíso, una tragedia amparada por el abandono de la ciudad (fuente: el autor).

\section{¿A QUÉ TIPO DE CONSTRUCCIONES SE PUEDE APLICAR?}

\section{las construcciones alude a soluciones}

individuales, o sea edificios y en este caso particular, las viviendas. El pensar que se pueden resolver los problemas de la ciudad a través de edificios puntuales, es una premisa desacertada e inmensamente arraigada en los arquitectos, no sólo en Chile, sino también en los países con una cultura más exigua en lo que respecta al diseño urbano y la planificación, en su mayoría, los países anglosajones. Nuestra tradición por otra parte, tiene más que ver con la cultura urbana europea, donde el urbanismo y la planificación son parte intrínseca de la cultura (o lo fueron en Chile hasta hace 40 años atrás), y donde las respuestas arquitectónicas responderían y se insertarían en una idea de ciudad. Dicho esto, el problema de Valparaíso no radica en la in-sustentabilidad de sus viviendas (imágenes 2 y 3), o en la a veces cuestionable calidad constructiva de éstas
(Sepúlveda, 2014), el problema radica en la espontaneidad y fortuita ubicación de ellas con respecto a la ciudad, la que en general, y más aún en los sectores afectados por el incendio, carecen de planificación urbana que organice su territorio con miras al bien colectivo de la ciudad y su población (imagen 4).

A modo de ilustración, me referiré también al caso del incendio en Victoria, Australia ("Black Saturday", 2009). Existen diferencias entre estos dos casos, las que se ilustran en la tabla 1. También similitudes importantes, tales como: las zonas afectadas eran en su mayoría pobladas por personas de bajos ingresos; muchos de los damnificados ya habian sufrido la pérdida de sus viviendas en incendios anteriores; limitada accesibilidad de los vehículos de emergencia (dispersión urbana en Victoria y deficiente accesibilidad en Valparaíso); en ambos casos se destaca la mala o inexistente planificación urbana como un factor importante, que contribuyó a la magnitud de la tragedia.
En Victoria, a pesar de las recomendaciones que respaldaban una mejora a la planificación, se optó por responder con viviendas sustentables, o sea, viviendas mejor equipadas para resistir incendios, pero desconectadas entre si y por consiguiente, perpetuando así su deficiente ubicación territorial (tabla 1). El énfasis en las viviendas, si bien respondió a las necesidades inmediatas de las personas afectadas, no resolvió el problema fundamental que era la deficiente ubicación de estas en el territorio, factor que influyó en la pérdida de vidas. Esto quiere decir que los habitantes de estas áreas en Victoria continúan expuestos a enfrentar la misma situación-sino peor, como algunos expertos auguran (Morton, 2009)-en el corto, mediano y largo plazo

En Australia, algunos expertos aseguran que existe una conexión entre el incendio de Victoria y el cambio climático, razón por la cual se cuestionó la lógica de reconstruir en los mismos lugares. Al 


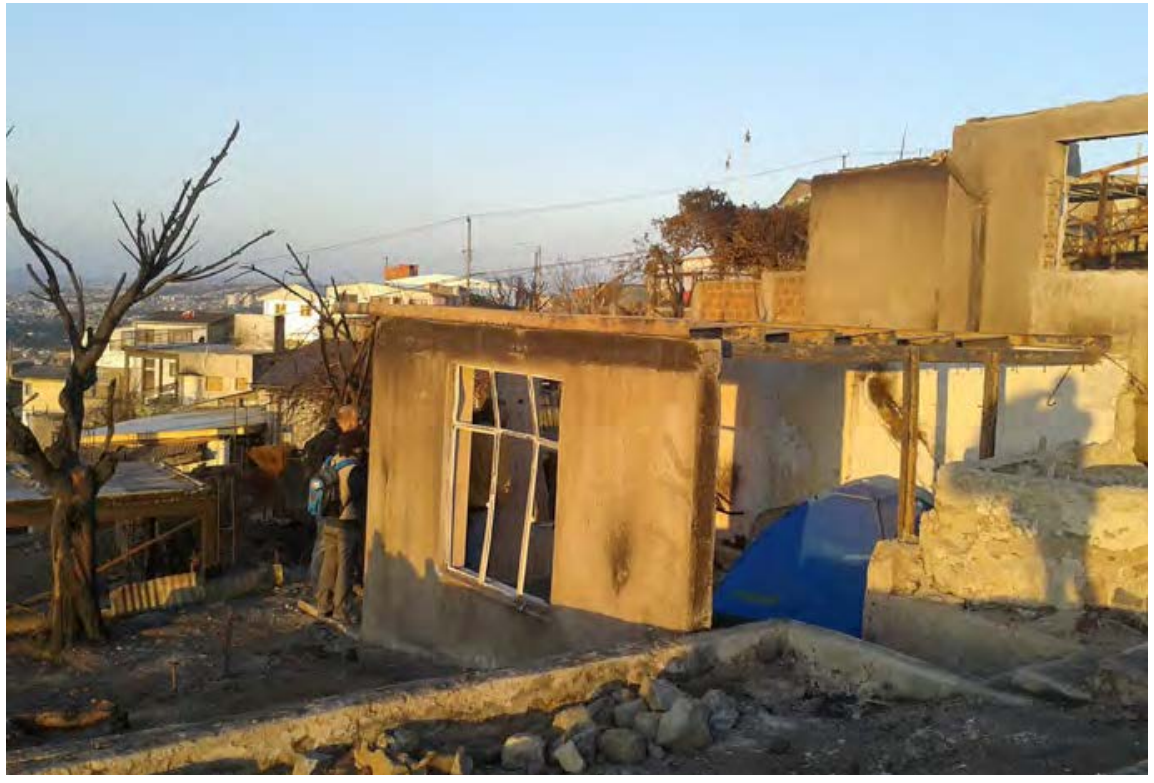

Imagen 2. Viviendas post incendio (fuente: Verónica Maturana).

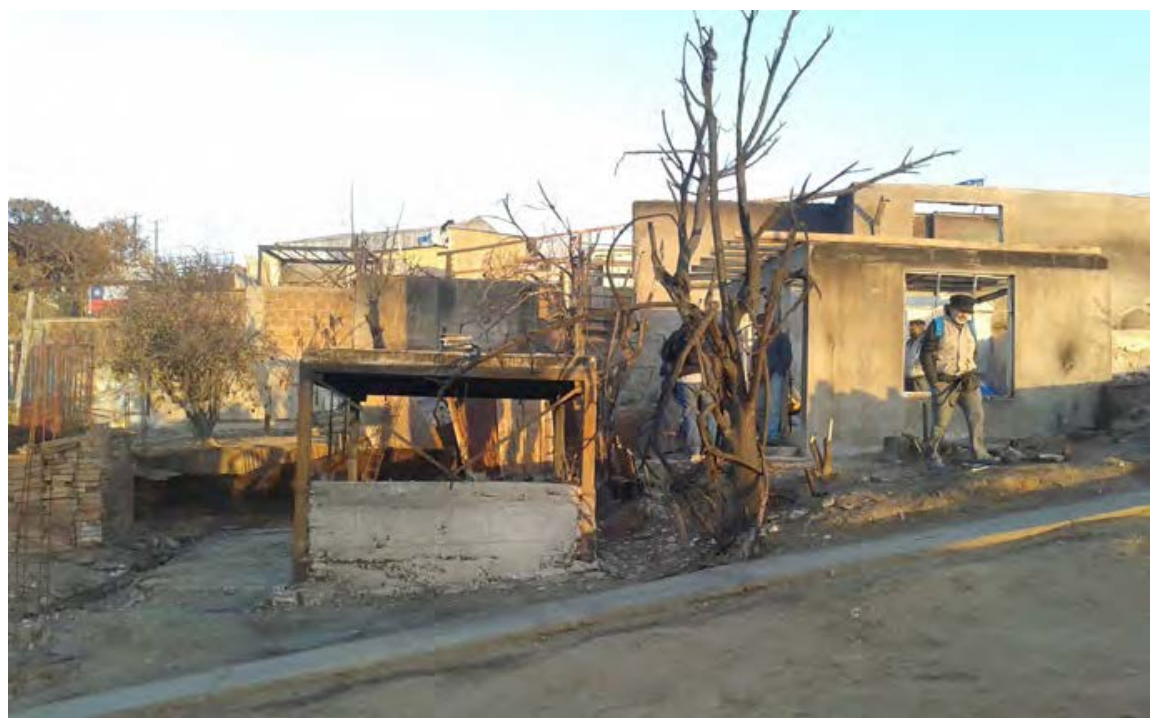

Imagen 3. Viviendas post incendio (fuente: Verónica Maturana).

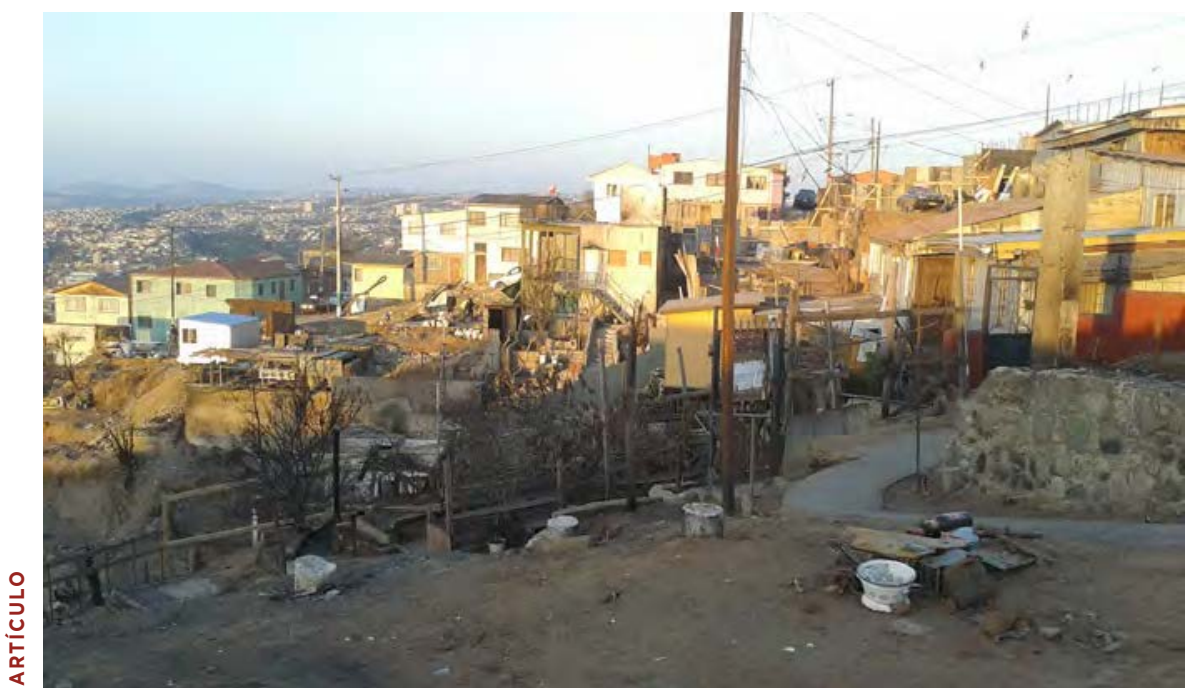

48 Imagen 4. Caminos de difícil acceso que imposibilitan la llegada de ayuda en casos de emergencia (fuente. Verónica Maturana). respecto, el climatologista de la Universidad de Melbourne, David Karoly, afirmó que "el riesgo de aumento en la intensidad y aumento en la frecuencia de estos incendios es real" (Morton, 2009). Si esto es efectivo, en Valparaíso no sólo debemos enfrentar el desafio de la planificación de la ciudad para proteger y generar mejoras medioambientales, sino también para prevenir lo que se prevé como una intensificación de las condiciones climáticas que generarían catástrofes de este tipo. Un estudio realizado por el académico de la Universidad de Chile, Miguel Castillo, confirma el aumento del número e intensidad de los incendios ocurridos desde el 2004, lo que atribuye en parte la expansión urbana en los cerros de Valparaíso (Fernández et al, 2014) Asimismo, ya en el 2012, el mismo académico alertaba de la "alta carga combustible acumulada" en los cerros de Valparaíso, situación exacerbada por los microbasurales y el difícil acceso (Arellano, 2014). El experto en incendios, Dr. Tolhurst, nos entrega una idea de la energía liberada por el incendio de Victoria, "equivalente a 1.500 bombas atómicas, del tamaño de aquellas usadas en Hiroshima, con la diferencia que esta energía fue liberada como una tormenta y no una explosión " (Hugues, 2009). Esta energía fue suficiente para haber proveído de energía al estado de Victoria (su industria y ciudades) por un año. Cabe preguntarse cuál fue la energía liberada en el incendio de Valparaíso y cuáles son los pronósticos, si a esta descontrolada expansión urbana se le suma el cambio climático.

\section{SUSTENTABILIDAD Y ESCALAS PERTINENTES DE APLICACIÓN:}

mportante aclarar y a veces cuestionar, e uso del concepto de sustentabilidad. En el caso de Valparaíso necesitamos distinguir la escala en la que la sustentabilidad tendrá el mayor y más pertinente impacto. 0 sea, si bien la noción de sustentabilidad es aplicable a todas las escalas, el énfasis y nivel directivo debe yacer donde se detecta el problema. Como diría Markku Lehtonen (2004), "el desarrollo sostenible depende de la capacidad de responder a los problemas sociales persistentes, que en alguna medida parecen haber sobrepasado los problemas medioambientales"

(Lehtonen, 2004). Las palabras de Lehtonen 
REVISTA AUS 16/Valparaíso, puerto y montaña: un desafí [en] pendiente/ Beatriz Maturana Cossio
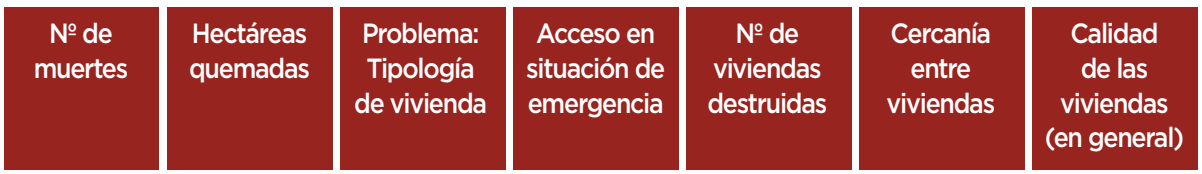

Problema:

Mejoras en Planificación planificación

\begin{tabular}{c|}
\hline $\begin{array}{c}\text { Victoria } \\
\text { Australia: } \\
\text { rural }\end{array}$ \\
\hline Valparaíso: \\
peri-urbana y \\
rural
\end{tabular}

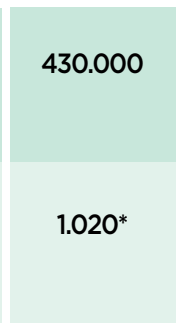

si

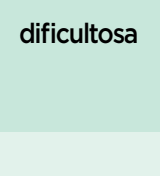

2030
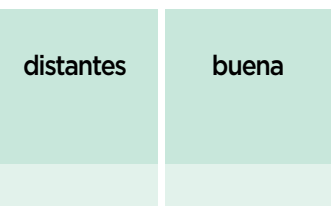

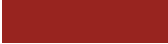

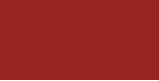

Tabla 1. Características comparativas entre el incendio de Victoria, Australia y Valparaiso, Chile (fuente: el autor).

* Otros datos indicarían que el número de hectáreas afectadas superan la cifra aqui señalada.

describen muy certeramente la situación de Valparaíso, donde existe un problema medioambiental, porque existe un problema social y económico persistente, el que no se resuelve con la aplicación de cambios a los inmuebles, convirtiéndolos en viviendas de adobe o bunkers de concreto para resistir el fuego (parte de las propuestas ofrecidas en Australia). El esporádico suministro de agua potable y la falta de alcantarillado que afectaba a los habitantes del cerro Rocuant, es indicativo del problema social, económico y ambiental que ya afectaba a gran parte de Valparaíso. Rocuant y otros cerros, habian sido identificados como zonas de peligro de incendio forestales por un estudio de la Conaf (Fernández et al., 2014) y era de conocimiento público que los microbasurales ubicados en las quebradas constituian un riesgo inminente. Si bien la materialidad y el diseño de los inmuebles son importantes, concuerdo con el arquitecto Daniel Morales quien afirma que "no puedes pensar solo el edificio sin contemplar lo que lo está rodeando" (Valencia, 2014).

Volviendo a esa tendencia por enfocarnos más en los edificios y menos en la ciudad, y a emular las experiencias extranjeras (que no es lo mismo que aprender de ellas), en un intento por hacer las ciudades más sustentables, se reconocieron en Australia dos factores de significativo impacto en la sustentabilidad de las ciudades: el gran

Imagen 5. Bunkers propuestos como solución para salvar vidas en el incendio de Victoria, Australia (fuente: Wildfire Safety Bunkers, http://www.wildfiresafetybunkers.com.au). tamaño de las viviendas y la baja densidad residencial-casas muy grandes y ciudades poco densas-(Blair et al., 2003). Ambos factores son escasamente relevantes a las ciudades chilenas, donde las viviendas (casas o departamentos) son pequeñas, el consumo energético, comparativamente hablando, es menor y la densidad es alta. Si bien, en este aspecto nos ubicamos en extremos opuestos, hay coincidencia en que tanto el problema de Valparaíso como el de Victoria, pasan por una adecuada planificación.

OTROS FUEGOS: Mencioné anteriormente la construcción de bunkers para referirme a algunas soluciones de emergencia propuestas para enfrentar los incendios en Victoria (imagen 5). Este concepto de bunker fue también incorporado por algunos arquitectos en las soluciones habitacionales para los damnificados, soluciones que en su mayoría desafían la estética tradicional de una vivienda y que además es económicamente inviable para los habitantes de esas áreas, quienes en su mayoría no podrían considerar los servicios de un arquitecto para el diseño de sus viviendas. Como señalado anteriormente, la tipología de asentamientos en Australia dista mucho de la tipología europea o la chilena, donde las últimas tienden a conglomerarse, con el caserío como su forma más básica de expresión. En Australia las viviendas, particularmente peri-urbanas y rurales, tienden a la dispersión, generando distancias infranqueables entre una casa y otra, lo que sumado a ubicación geográfica en áreas de reconocido riesgo, generó uno de los mayores impedimentos al tratar de salvar vidas y viviendas. Otra característica de la tragedia en Victoria fue que la mayoría de los afectados eran familias de clase media baja, baja y de sectores vulnerables de la población, los que como en Chile, buscan construir en terrenos de menor costo y frecuentemente inapropiados para habitar.

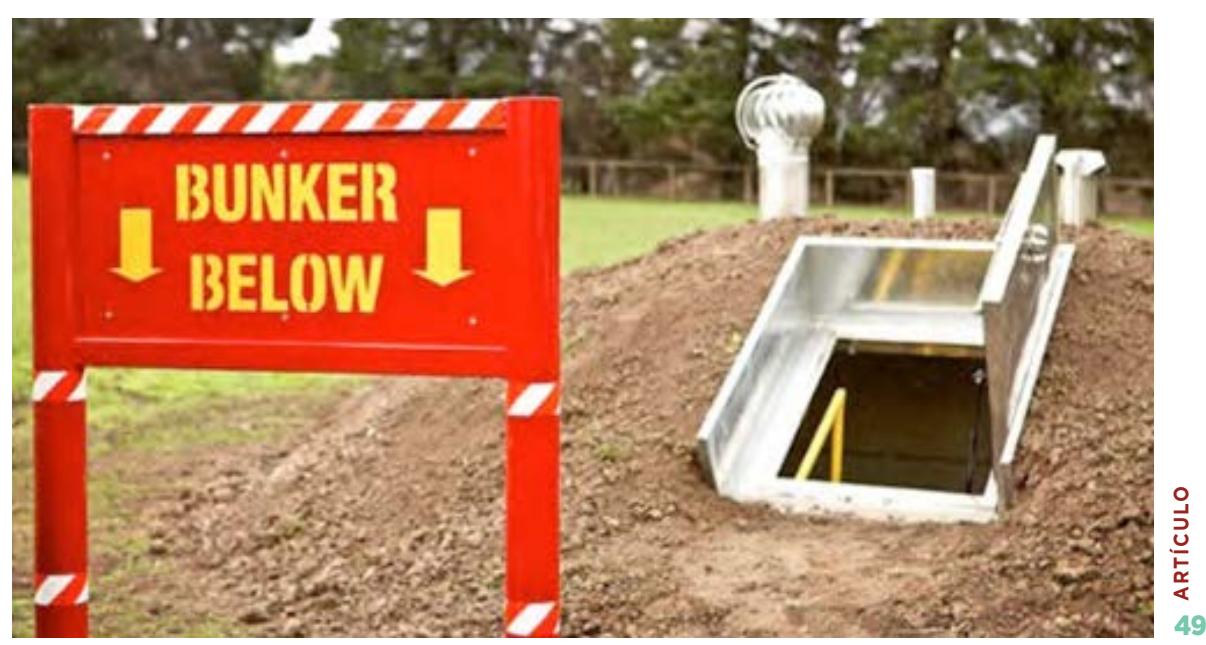


En Victoria se discutieron dos frentes de acción para enfrentar la tragedia, la reconstrucción individual de las viviendas (pasando por una etapa de vivienda de emergencia y luego las viviendas definitivas) y la posibilidad de repensar y planificar (por primera vez) la forma de estos asentamientos. Sin embargo, y a pesar de los numerosos estudios que coincidían en la importancia de la planificación, se optó como por inercia por la reconstrucción y sustentabilidad aplicada solamente a las viviendas. Estos dos frentes, la reconstrucción de viviendas individuales y la planificación, son compartidos también en las discusiones y propuestas para Valparaíso.

El incendio en Victoria dio pie a numerosas propuestas por firmas de arquitectos, también a muchos concursos y propuestas realizadas por estudiantes de arquitectura. Estas propuestas, que en general se enfocaban en la vivienda, fueron en su mayoría descartadas por los habitantes, ya que no eran económicamente factibles y no respondían a sus necesidades, tanto físicas como emocionales. Los habitantes no buscaban una vivienda icónica (ya sea en su forma o función), sino una vivienda típica, que no los distinguiera del otro. Factores tales como que muchos de estos habitantes no tenían seguros contra incendios exacerbó la necesidad de viviendas inmediatas y accesibles económicamente, lo que reforzó el ya estable dominio del mercado inmobiliario. Siendo la vivienda una necesidad prioritaria, estas se reconstruyeron sin mediar un plan maestro (Maturana, 2009). Si bien se introdujeron algunas normativas específicas, como por ejemplo con respecto al diseño de techos, puertas y ventanas, esta no es la escala de intervención que pudiera proteger a Victoria de una nueva tragedia como la ocurrida en el 2009. En suma, sin un liderazgo por parte del gobierno, en Victoria se reconstruyó "ladrido a ladrillo"-palabras textuales del entonces Primer Ministro Kevin Rudd (Nicholson, 2009)-, obviando la urgente necesidad de planificación que había sido reconocida como uno de los mayores problemas a la hora de salvar vidas e infraestructura.

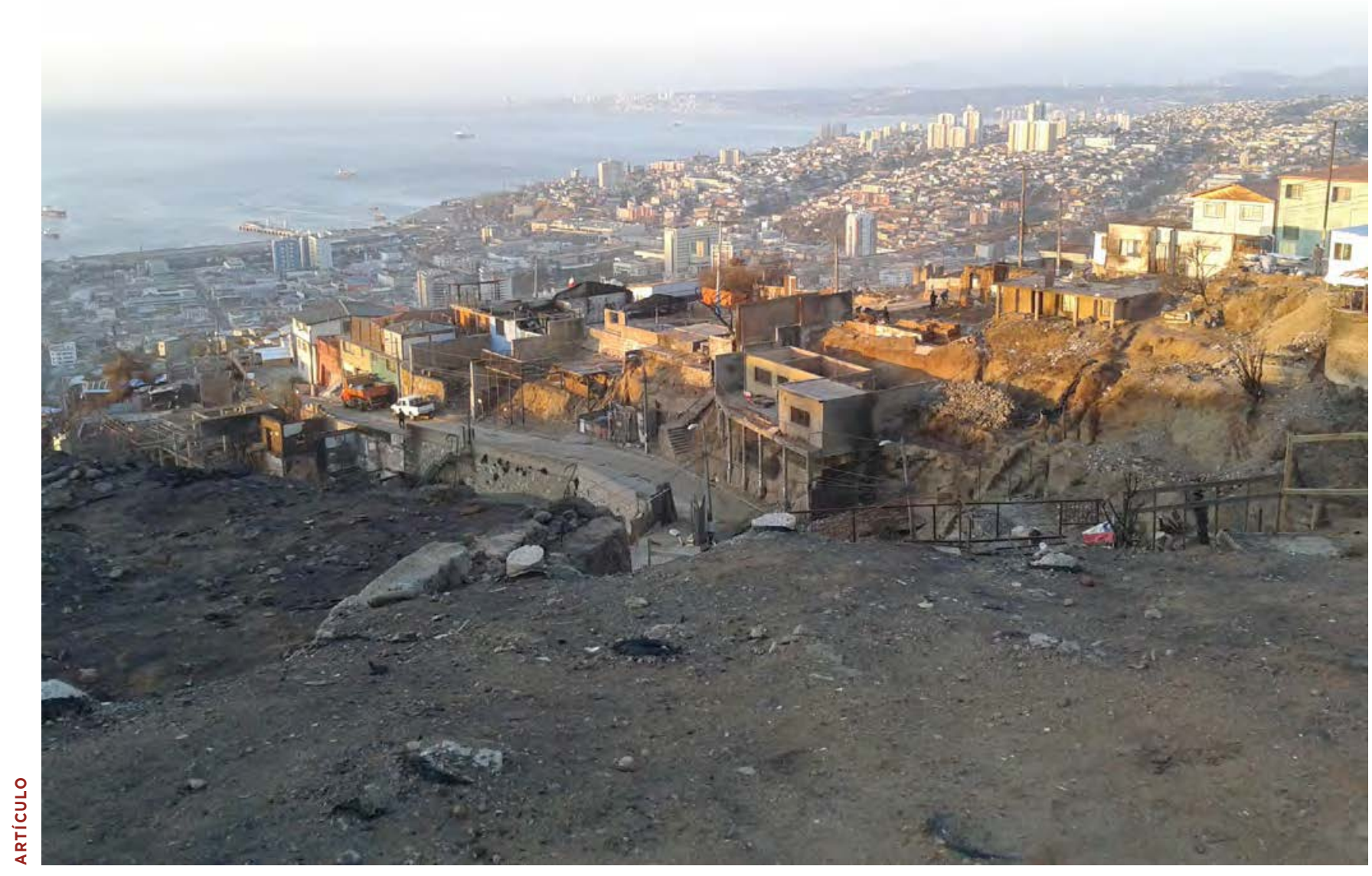

50 Imagen 6. Valparaíso, una geografía compleja que refleja la inequidad social (fuente: Verónica Maturana). 


\section{LA CULTURA URBANA DE LA PRECARIEDAD PUEDE SER SUPERADA.}

En Valparaíso, muchas de las viviendas incendiadas habían sido construidas dando respuesta a las condiciones físicas, económicas y sociales del entorno más inmediato, viviendas que si bien pueden ser sólidas, en su mayoría carecen de calidad. A esta mayoritariamente precariedad y abandono (Fernández et al., 2014), se la ha confundido con cultura urbana. De una forma u otra, estas viviendas individuales, conjuntos mal planificados o sin planificación, terminan conformando una ciudad también precaria-no la ciudad de calidad a la que aspiramos, al menos no para todos-.

Tanto en Victoria como en Valparaíso, el paisaje y la belleza de estas zonas han sido admirado desde la lejanía-la lejanía del turismo o la lejanía física desde donde se los contempla-. Pese a que las montañas, o cerros en el caso de Valparaíso, conforman desde lejos un atractivo palimpsesto de colores, no es allí, en los cerros más afectados por el incendio, donde se albergan los turistas, y no es alli donde se generan y disfrutan los ingresos y las oportunidades económicas (imagen 6). Si la belleza de Valparaíso y su valor se debe en gran parte a su geografía equitativamente distribuida a lo largo de esta ciudad, ¿qué impide que este valor no se transforme en ingresos y calidad medioambiental para toda la ciudad? Es en esta forma espontánea y desentendida de las necesidades de las mayorías que viven en sus montañas, que se evidencia el abandono, un dejar de hacer que contradice la aspiración de sustentabilidad, la que requiere un activo concebir, hacer y construir un medioambiente sano y de calidad.

Hay otras dos preguntas a las que creo haberme referido ya de alguna manera, estas son ¿Cómo se puede ejecutar (requerimientos, condiciones, estrategias)? ¿Cuáles son sus ventajas y dificultades?

Me quedaré con la ejecución, donde es importante identificar el nivel de acción: primeramente la emergencia y luego la solución estratégica de ésta. Digo estratégica porque debemos evitar crear falsas expectativas, que sean contraproducentes al bienestar de las personas en el mediano y largo plazo -importante también, porque las soluciones de emergencia tienden a ser permanentes y a delinear la ciudad en formas no previstas-. La planificación para la emergencia pasa por una evaluación rigurosa y la identificación de los lugares que no podrán ser rehabilitados y por lo tanto donde tampoco se podrían ubicar las viviendas de emergencia, evitando así falsas ilusiones. También, como dicho anteriormente, se debe incorporar la revisión y mejoramiento del plan de desarrollo urbano (el que se supondría existente) Responder a la emergencia es un trabajo complejo y delicado porque no se trata solamente de levantar un techo, ya que aquí estamos hablando de arraigo, educación y eficiencia económica que permita resolver la emergencia, sin entorpecer las soluciones definitivas y sustentables en el largo plazo.

Valparaiso ha estado en llamas por décadas, social, económica y medio ambientalmente hablando. Esta situación no debe ser mantenida, tampoco romantizada en nombre de la cultura, entendiendo que la cultura es lo que hacemos y no lo que dejamos de hacer.

La sustentabilidad expresada en bunkers de concreto o en casas con materiales reciclados, adobe $u$ otro, puede responder a la inmediatez de la catástrofe, también a las necesidades emocionales que situaciones como estas despiertan, sin embargo no responden a la escala donde se genera el problema y por ende donde radica la solución. Valparaíso, ciudad de geografía compleja, de borde costero y montañas, necesita una planificación urbana que reconozca las riquezas y desafíos que ofrece su geografía en pos de una ciudad de calidad medioambiental para todos. Como se dijo en relación a Victoria, Australia, y hoy pertinente a Valparaíso, la devastación se nos presenta con una sombría oportunidad de reescribir cómo hacemos ciudad y considerar nuevas estrategias (McLaren, 2009). $\Delta \mathbf{0 0}$

\section{REFERENCIAS}

Arellano, A., Bezama, B., 2014. El historial de negligencia y corrupción que hizo arder a Valparaíso. Santiago: CIPER Centro de Investigación Periodistica, Chile

Blair, J., Fisher, M., Prasad, D., Judd, B., Soebarto, V., Hyde, R., Zehner, R., 2003. Affordability and Sustainability Outcomes of 'Greenfield' Suburban Development and Master Planned Communities - a case study approach using triple bottom line assessment. Australian Housing and Urban Research Institute UNSW - UWS Research Centre. http://www.ahuri.edu.au/attachments/70137_pp_greenfield.pdf

Fernández, B., Urquieta, C., y Sepúlveda, N., 2014. Los informes que advirtieron sobre los riesgos de incendio en Valparaiso y que nadie escuchó. El Mostrador. http://www.elmostrador.cl/pais/2014/04/15/los-informes-queadvirtieron-sobre-los-riesgos-de-incendio-en-valparaiso-y-que-nadie-escucho/

Higueras, E., 2009. Ordenación del territorio. Planificación ambiental y urbanismo bioclimático (1 ed. Vol. 1): Dapp.

Hugues, G., 2009. Black Saturday fires had power of 1500 A-bombs. The Australian. Retrieved from http://www.theaustralian.com.au/news/nation/black-saturday-fires-had-power-of-1500-a-bombs/story-

e6frg6nf-1225714533980

Lehtonen, M., 2004. The environmental-social interface of sustainable development: capabilities, social capital, institutions. Ecological economics, 49(2), 199-214.

Martinez, K., 2011. Comunidades y Barrios Sustentables: Sistemas de Certificación Avanzando hacia la Sustentabilidad de la Escala Urbana Intermedia. Revista AUS № 10, pp. 18-21.

Maturana, B. C. 2009. Victoria's Bushfires: Time to Reflect New Urban Strategies. Planning News, pp. 12-13.

McLaren, O., 2009. Bushfire Tragedy Rewrites Rules for Architects. ABC News.

MINVU. (Mayo 13, 2014). Ministra Saball anunció Plan de Reconstrucción de viviendas para Valparaíso. Santiago: MINVU Retrieved from http://www.minvu.cl/opensite_det_20140513154006.aspx

Morton, A. 2009. Climate change must be 'a factor' in deciding whether to rebuild. The Age. Retrieved from http://www.watoday.com.au/national/climate-change-must-be-a-factor-in-deciding-whether-to-rebuild-

20090210-8315.html?page=-1

Nicholson, B., Rood, D., 2009. We'll rebuild brick by brick. The Age. Retrieved from http://www.theage.com.au/national/well-rebuild-brick-by-brick-20090210-83k9.html?page=-1

Sepúlveda, N., 2014. El incendio es resultado de una completa irresponsabilidad que se ha ido acumulando con el paso de los años. El Mostrador. http://www.elmostrador.cl/pais/2014/04/14/el-incendio-es-resultado-de-unacompleta-irresponsabilidad-que-se-ha-ido-acumulando-con-el-paso-de-los-anos/

Valencia, N., 2014. Incendio en Valparaiso: Diez dilemas ante la reconstrucción. Plataforma Arquitectura. http://www.plataformaarquitectura.cl/cl/02-353492/incendio-en-valparaiso-diez-dilemas-ante-la-reconstruccion Valencia, N., 2014. A un mes del incendio, arquitectos Daniel Morales y Marcelo Ruiz Fernández: "Valparaíso tocó fondo". Plataforma Arquitectura. http://www.archdaily.mx/mx/02-361038/a-un-mes-del-incendio-arquitectosdaniel-morales-y-marcelo-ruiz-fernandez-valparaiso-toco-fondo 